\title{
Enhancement in nutrient accumulation and growth of oil palm seedlings caused by PGPR under field nursery conditions
}

\begin{abstract}
Plant growth promoting rhizobacteria (PGPR) (e.g., Azospirillum and Bacillus spp.) have been reported to enhance growth and fix N2 with several nonleguminous crops. These rhizobacteria have the potential to be applied to oil palm seedlings and, consequently, reduce the cost of nitrogenous fertilizer. The rhizobacteria are also known as a bioenhancer for the ability to increase root growth and enhanced water and nutrient absorption by the host plants. An experiment was carried out in the field nursery station, Federal Land Development Authorities (FELDA), Bukit Mendi, Pahang, Malaysia, to observe the effects of PGPR inoculation on enhanced nutrient accumulation and plant growth (tops and roots) of oil palm seedlings under field nursery conditions. The inoculation process showed positive response in enhancing higher accumulation of nitrogen $(\mathrm{N})$, phosphorus $(\mathrm{P})$, and potassium $(\mathrm{K})$ in the plant tissues, enhanced root dry weight and top growth (dry matter and leaf chlorophyll content) of the host plants under field nursery conditions. Copyright $($ ) Taylor \& Francis, Inc.
\end{abstract}

Keyword: Field nursery; Growth; Nutrient accumulation; Oil palm seedlings; PGPR 